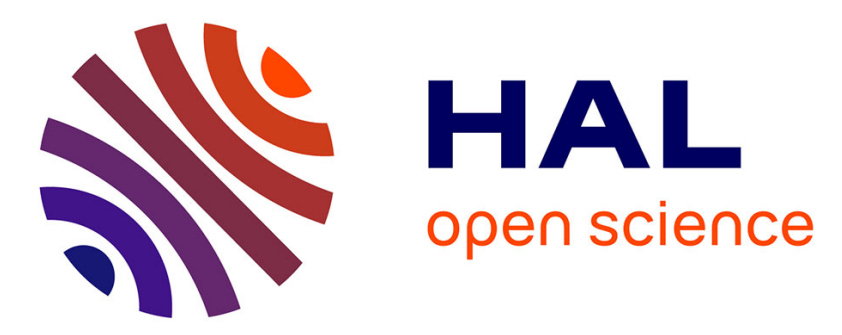

\title{
Infrared thermography applied to the analysis of material behavior: a brief overview
}

André Chrysochoos

\section{To cite this version:}

André Chrysochoos. Infrared thermography applied to the analysis of material behavior: a brief overview. Quantitative InfraRed Thermography Journal, 2012, 9 (2), pp.193-208. 10.1080/17686733.2012.746069 . hal-00832341

\section{HAL Id: hal-00832341 https://hal.science/hal-00832341}

Submitted on 10 Jun 2013

HAL is a multi-disciplinary open access archive for the deposit and dissemination of scientific research documents, whether they are published or not. The documents may come from teaching and research institutions in France or abroad, or from public or private research centers.
L'archive ouverte pluridisciplinaire HAL, est destinée au dépôt et à la diffusion de documents scientifiques de niveau recherche, publiés ou non, émanant des établissements d'enseignement et de recherche français ou étrangers, des laboratoires publics ou privés. 
Infrared thermography applied to the analysis of material behavior: a brief overview

André Chrysochoos

Laboratory of Mechanics and Civil Engineering, CC 048, Montpellier University,

Place E. Bataillon, 34095 Montpellier Cedex 05, France

(andre.chrysochoos@univ-montp2.fr) 


\section{Infrared thermography applied to the analysis of material behavior: a brief overview}

The characterization of material behavior under various loading conditions involves two closely related aspects, namely mechanical and thermal, that can be grouped into one general thermomechanical framework. The goal of this paper is to briefly review specific applications of infrared techniques, over a range of loading conditions, such as quasi-static and polycyclic, for various types of material. Some experimental and theoretical issues are discussed to highlight the additional knowledge that can be gained through the application of these techniques to the mechanical characterization of materials.

Keywords: infrared thermography, material behavior, dissipation, thermomechanical couplings.

\section{Introduction}

The use of infrared techniques in Mechanics of Materials and Structures has been considerably extended over the last 20 years in the academic laboratories and industrial research and development centers. This change was facilitated by the progress achieved in signal digitization, data transfer rates and image processing techniques, leading to more user-friendly thermographic workstations.

The major asset of thermographic systems is that they provide contact-less fullfield measurements of surface temperature. Besides, current IR cameras allow storage of thermal images of about 80000 pixels at frame rates reaching up to several hundred Hertz, and beyond under certain conditions. The new sensors allow estimation of temperature variations from several hundredths to several hundreds of Celsius degrees. Finally, some electronic systems enable a reduction in the size of thermal images, thereby considerably increasing the framing rate. The latter capability is particularly valuable for studying the dynamic transient behavior of materials. 
It was only at the end of the 1970s that infrared techniques were first used to obtain quantitative results in non-military mechanical applications. The first applications to the mechanics of materials were directed towards non-destructive testing. The oldest application domain is certainly thermoelastic stress analysis which considers the thermo-dilatability of materials [1,2]. Under adiabatic conditions, thermoelastic stress analysis (TSA) provides a direct link between temperature variations and hydrostatic stress variation patterns. TSA has given rise to a wide range of applications $[3,4]$. The « Stress Pattern Analysis by Thermal Emission (SPATE) was the standard reference system for a long time. In the last 20 years, this system has been used by over 150 research groups in many application fields: automotive, aeronautics, naval design, biomechanics, etc. However, since SPATE used a unique infrared element, the construction of full-field measurements required delicate optical systems (rotating mirrors) and reconstruction of the stress pattern was quite time consuming. In the last 10 years or so, these drawbacks have prompted some laboratories or companies to develop modern versions of SPATE using infrared focal plane array cameras (IRFPA).

Another expanding application domain for infrared techniques concerns material and structural fatigue. For quasi-static cyclic loading, the self-heating induced by fatigue spans from several degrees Celsius to several dozens of degrees [5-8]. However, dynamic fatigue involves loading frequencies of about several dozens of $\mathrm{kHz}$ (e.g. 24 $\mathrm{kHz}$ ), so self-heating increases up to several hundreds of degrees depending on the material properties and heat exchange intensity [9]. The first studies of thermal effects in fatigue were performed using infrared pyrometers or monodetector cameras. They gave rise to a simple method to rapidly estimate the fatigue limit of materials $[5,10]$. This method considers the mean temperature of the part under investigation subjected to 
a series of blocks of cycles with an increasing stress range. The block duration is long enough to observe stabilization of the mean temperature. This stabilization derives from the equilibrium between the mean dissipated energy rate and heat exchanges between the part and its surroundings. The fatigue limit is thus empirically related to the stress range from which the mean stabilized temperature (as a function of the stress range) drastically increases. This method often gave interesting results, as discussed in [11]. Since the advent of IRFPA cameras, another method $[12,13]$, used at the end of the seventies, was adopted by some camera designers. This method is based on timefrequency analysis of the thermal signal, and assumes that mechanical energy dissipation during monochromatic cyclic loading can be detected by estimating the signal intensity at $2 f$, where $f$ is the loading frequency.

Applications of IR techniques in mechanics of materials where the temperature variations reach several hundreds of degrees Celsius should also be mentioned. Tests performed to study the dynamic behavior of materials, some high speed machining or forming process belong to this class of applications where self-heating is very substantial $[14,15]$. Naturally, welding operations and tribology tests also involve large temperature variations.

\section{From thermal to calorimetric analysis of material behavior}

As previously highlighted, the deformation of solid materials is then nearly always accompanied by temperature variations. These variations are induced by heat sources of a range of different natures and that vary in their intensity and spatial distribution. They also depend on the diffusivity of the material and the boundary conditions, which implies that the thermal information is not completely intrinsic to the material behavior. Besides, temperature variations induced in solid materials by quasi-static mechanical loadings may remain low and are still often neglected. Consequently, from a modeling 
standpoint, these experimental facts implicitly inspired the wide development of isothermal models that have been documented in the literature. Nevertheless, temperature variations, no matter how small they are, may correspond to amounts of heat that cannot be neglected in the energy balance, and should consequently be taken into account in the thermomechanical modeling. Mandel and Bui were among the first to show the great benefits of combining the mechanical and energy aspects of the same phenomena interpreted within a thermodynamic framework $[16,17]$.

Over the last two decades, a few research groups have therefore developed infrared techniques in an effort to identify heat source distributions from surface temperature fields during a deformation process. It is worth noting that the nature of heat sources highly depends on the material behavior. They may represent an intrinsic dissipation of energy (plasticity, viscosity, damage, etc.), or result from thermomechanical coupling mechanisms (thermoelasticity, rubber elasticity, latent heat of solid-solid phase transition, etc.). At this point, it should be stressed that calorimetric information associated with material behavior is still rarely available in the literature. This kind of information is, however, of great interest in that it characterizes the irreversibility of deformation mechanisms and reveals the thermal sensitivity of the material. From a modeling point of view, coupling sources and dissipation are respectively related to state and evolution constitutive equations, and consequently provide useful calorimetric safeguards to check the consistency of (thermo) mechanical models.

In what follows, a brief review of the theoretical background used to define strain-induced heat sources and the whole energy balance is given. The different devices involved in the setup and experimental procedure developed for quasi-static loading conditions are then described. Finally, two examples dealing with thermal and 
calorimetric results induced by material deformation are presented. The first one focuses on polymer materials. The second underlines the interest of thermal and calorimetric analysis of high cycle fatigue mechanisms of metallic materials. In both cases, the crucial consequences of experimental findings in terms of understanding and modeling of the material behavior are discussed.

\subsection{Some words about the theoretical framework}

Concepts and results of the thermodynamics of irreversible processes must be used to define the different heat sources induced by deformation processes. In this paper, the thermodynamics with internal state variables will be used, whereby the equilibrium state of each volume material element is characterized by a finite set of $n$ state variables. The chosen state variables are the absolute temperature $T$, the strain tensor $\varepsilon$, and the $n$ 2 scalar components $\left(\alpha_{1}, \ldots, \alpha_{n-2}\right)$ of the vector $\alpha$ of so-called "internal" variables that model the micro-structural state of the material. By construction, the thermodynamic potential is the Helmholtz free energy $\psi$. Combining the first and second principles of thermodynamics leads to the local heat diffusion equation:

$$
\rho C \dot{T}-\operatorname{div}(k \operatorname{grad} T)=\underbrace{\sigma: D-\rho \psi_{, \varepsilon}: \dot{\varepsilon}-\rho \psi_{, \alpha} . \dot{\alpha}}_{d_{1}}+\underbrace{\rho T \psi_{, \varepsilon T}: \dot{\varepsilon}+\rho T \psi_{, \alpha T} \cdot \dot{\alpha}}_{w_{\text {thm }}^{\circ}}+r_{\mathrm{e}}
$$

where $\rho$ denotes the mass density, $C$ the specific heat, $k$ the heat conduction tensor, $\sigma$ the Cauchy stress tensor and $D$ the Eulerian strain rate tensor. The left-hand side conventionally consists of a differential operator applied to the temperature, while the right-hand side pools the various types of heat source: intrinsic dissipation $d_{1}$, thermomechanical coupling sources, and the possible external heat supply $r_{e}$ (e.g. radiation exchanges). Intrinsic dissipation characterizes the material degradation accompanying the irreversible transformation of the microstructure, while the thermo-mechanical heat 
sources reflect the thermo-sensitivity of the matter, indicating that mechanical and thermal states are closely coupled. The non-standard dot $\left(w_{t h m}\right)^{\circ}$ specifies that the heat rate is thermodynamic path dependent (i.e. $w_{t h m}$ is not, a priori, a state function).

Note that the heat diffusion equation is a partial differential equation applied to temperature. Estimating the left hand side using the thermal data then leads to an overall estimate of the different heat sources grouped in the right hand side. Note also that each differential operator is weighted by material parameters such as $\rho, C$ and $k$. So, whatever the method used to determine the heat source field, the reliability of the results depends not only on the accuracy of the temperature measurements [18] and of the numerical methods used to estimate the differential operators but also on the knowledge of the material parameters [19].

So far, the following strong hypotheses have been put forward during infrared image processing to compute the heat sources:

- Mass density and specific heat are material constants, independent of the thermodynamic state.

- The heat conduction tensor remains constant and isotropic during the test $\left(k_{\mathrm{ij}}=\right.$ $\left.k . \delta_{\mathrm{ij}}\right)$.

- The external heat supply $r_{\mathrm{e}}$ due to heat exchange by radiation is timeindependent, so the equilibrium temperature field $T_{0}$ verifies $-\Delta T_{0}=r_{\mathrm{e}}$. It is then convenient to consider the temperature variation $\theta$ defined by $\theta=T-T_{0}$.

Taking into account these latter hypotheses, the heat equation can be rewritten in the following compact form:

$$
\rho C d \theta / d t-\operatorname{div}(k \operatorname{grad} \theta)=w_{\mathrm{h}}^{\circ},
$$


where $w_{\mathrm{h}}^{\mathrm{o}}$ stands for the overall heat source, with $w_{\mathrm{h}}$ being the locally generated heat evolved.

These hypotheses are reasonable for many situations. However, they are inadequate when a strong anisotropy pre-exists or develops during strain, when strain and/or damage localization occurs or when the thermo-mechanical loading leads to dynamic instabilities. For such fully realistic situations, alternative hypotheses have to be proposed in order to guarantee a reliable calorimetric analysis of the mechanical behavior. At this stage, let us note that the measurable quantity in a typical experiment is the evolution of the thermal field $\theta$ in the deformed solid, at various spatial resolution levels, even to a single point measurement.

\subsection{Experimental setup}

Figure 1 illustrates a typical experimental set-up designed for quasi-static tests. It involves a MTS hydraulic testing machine (frame: $100 \mathrm{kN}$, load cell: $25 \mathrm{kN}$ ), a Cedip Jade III infrared camera or a Cedip Titanium camera. The optical axis of the camera was set perpendicularly to the frame of the testing machine, and it remained fixed during the test.
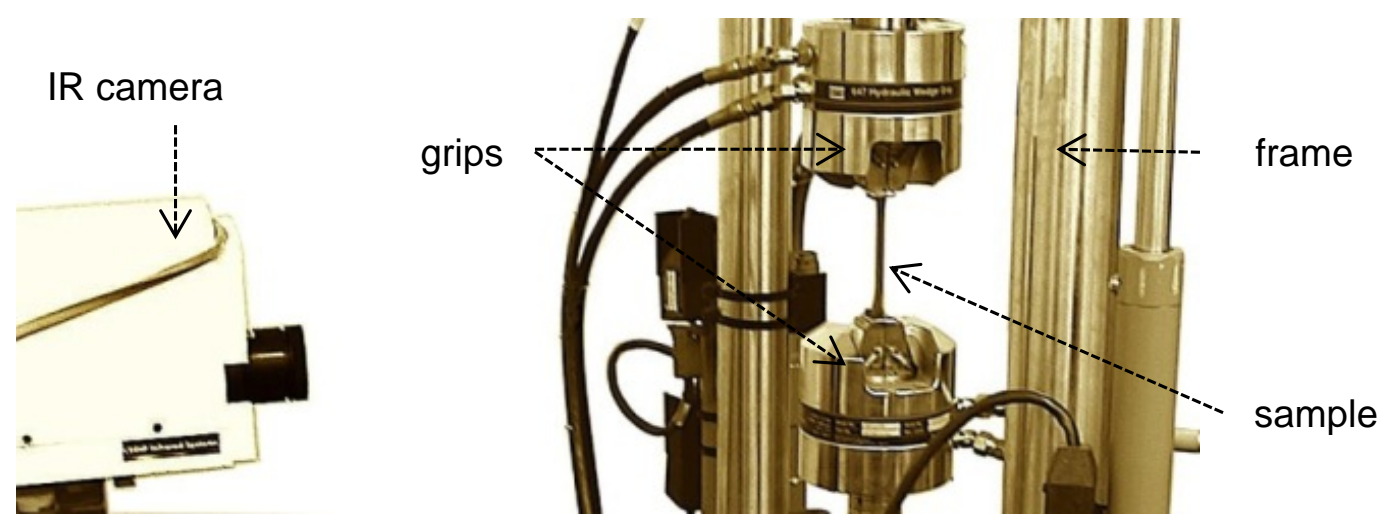

Figure 1. Experimental arrangement for quasi-static tests 
The main characteristics of IR cameras are given in Table 1. Note that, for large deformation processes, the IR camera can be successfully associated with a visible CCD camera in order to track the temperature of material surface elements using, for instance, speckle image correlation (Chrysochoos et al., 09), (Wattrisse et al., 01).

Table 1 Main camera characteristics

\begin{tabular}{lccc}
\hline & $\begin{array}{c}\text { image } \\
\text { size (pix. })\end{array}$ & $\begin{array}{c}\text { scale factor } \\
\left(\mu \text { m.pix. }^{-1}\right)\end{array}$ & $\begin{array}{c}\text { frame rate } \\
\end{array}$ \\
& $320 \times 240$ & 524 & 25 \\
Cedip Jade III & $640 \times 480$ & 524 & 300 \\
Cedip Titanium & & & \\
\hline
\end{tabular}

\subsubsection{Pixel calibration of IRFPA cameras}

An IRFPA camera, like any IR sensor, is sensitive to thermal radiation emitted by the region that it targets. When the target can be considered as a black-body, the thermal radiation energy can be directly related to the temperature of the targeted surface. The calibration stage consists of establishing the relationship between the digitized IR influx and the temperature. It is thus of major importance, particularly when thermal data have to be used quantitatively to derive heat sources. A basic sketch of the thermography workstation is shown in Figure 2. The IR influx passing through the lens is converted into an electrical tension signal by the detector. An analog-to-digital converter (ADC) then provides a digital signal coded in digital levels (DL). 


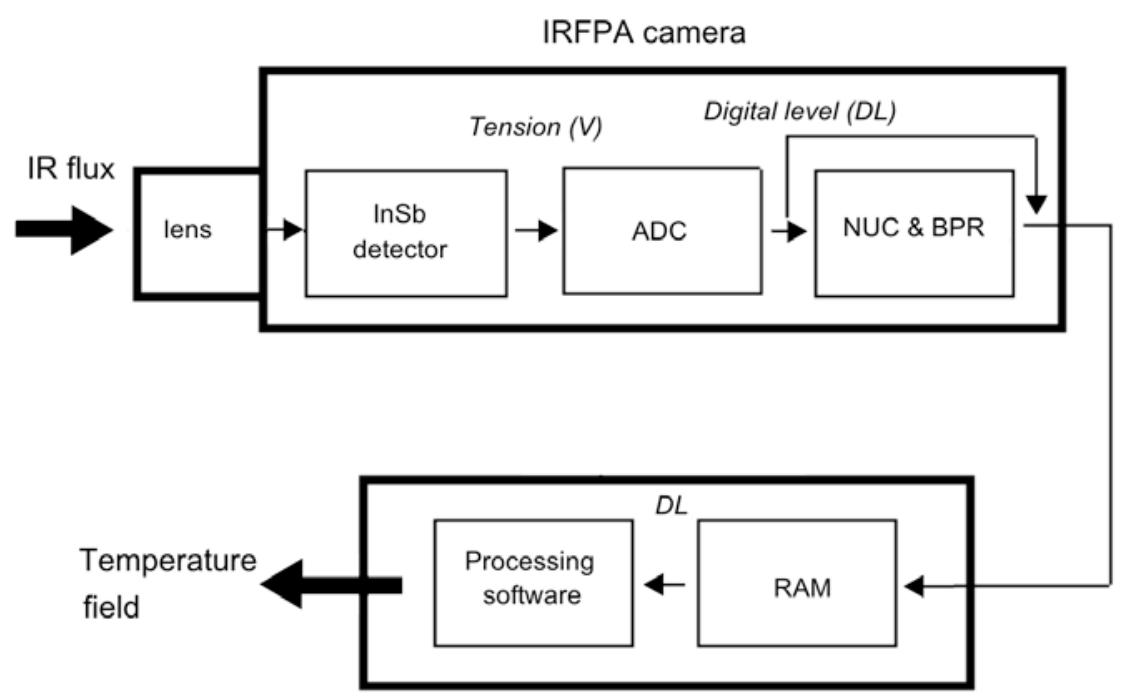

Processing software

Figure 2. Basic sketch of the thermography workstation

The next stage takes into account the non-uniform response of the detector elements when placed in front of a uniform radiative source. This is the non-uniformity correction (NUC) operation. At this stage, so-called "bad pixels" are also tracked and replaced. This is the bad pixel replacement (BPR) operation. These basic operations are part of the standard calibration protocol. The digital video is finally stored in the RAM of the computer. A software package developed by the camera designer then enables calibration, processing and visualization of thermal data.

A stable and spatially uniform IR radiation source is used to perform NUC, BPR and camera signal calibration. For example, a planar extended area blackbody SR 804A (by CI Systems) having the following main specifications has been used in our application: black body surface accuracy (aperture: $4 " \times 4 ",+/-0.008^{\circ} \mathrm{C}$ for $5^{\circ} \mathrm{C}$ temperature amplitude), thermal resolution $\left(\delta T_{R}=0.01^{\circ} \mathrm{C}\right)$, and spatial non-uniformity of the target (less than $+/-0.02^{\circ} \mathrm{C}$ ). 
Signal standard calibration can be carried out once the NUC and BPR operations are performed. This calibration should theoretically be based on Planck's law. Indeed, for a quasi-monochromatic detector placed in front of a uniform black body at temperature $T$, Planck's law is used to derive the following expression of the camera signal $S$ [22]:

$$
S=\gamma \frac{C_{1} \lambda^{-5}}{\exp \left(\frac{C_{2}}{\lambda T}\right)-1}+U_{0}
$$

where $\lambda$ is the mean wavelength of the spectral range of the detector, while $C_{1}, C_{2}, \gamma$ and $U_{0}$ are constants. Designers and offices of standardization and metrology generally prefer simplified versions of Equation (3). They also often choose a simple fitting of the curve $S(T)$ by a polynomial of degree 2 or 3 , especially when small thermal ranges $\left[T_{1}\right.$, $\left.T_{2}\right]$ are considered.

$$
S(T)=\sum_{p=0}^{P} a_{p} T^{p}, P=2 \text { or } 3, T \in\left[T_{1}, T_{2}\right]
$$

The polynomial coefficients $\left(a_{p}, p=0,1, \ldots, P\right)$ are classically derived from a least-squares fit of $\left(S_{j}, T_{j}\right)$ couples $(j=1, . ., J)$. The number $J$ of thermal equilibrium states must be much greater than $P$. Besides, the temperatures $T_{j}$ have to be regularly distributed within $\left[T_{1}, T_{2}\right]$ to get a reliable fit.

The major interest of the standard calibration procedure described above is, in our opinion, its simplicity of use. The NUC table (gain + offset tables), the bad pixel list and the coefficients of the calibration law can be automatically managed by software. Different calibration files can be saved, corresponding to different parameter sets of the camera (frame size, integration time, optional filter, etc.). Nevertheless, this calibration 
is based on homogenization of the detector response, whereas pixel responses are generally heterogeneous. The goal is to get a uniform signal of the detector elements when the detector is placed in front of a uniform source. This enables the user to identify a single calibration law inasmuch as $" i=1,2, \ldots, I$, with $I$ being the number of matrix elements.

We have already underlined in [23] that the NUC operation supposes a linear response of each detector element. First, this condition may limit the thermal range used for the calibration or may induce uncontrolled errors if some elements start to behave nonlinearly. Secondly, even if all element responses remain linear, the standard calibration protocol replaces some bad pixels because their responses are far from the mean detector response. In the framework of thermomechanical applications, this replacement operation may distort the thermal gradient computation by spatially correlating measurement errors. This distortion consequently affects the heat source estimate.

It is fortunately possible to shortcut NUC and BPR operations with some IR thermography workstation (e.g. Cedip cameras). This feature was used to develop an individual pixel calibration based on polynomial fitting of the digital level $s_{\mathrm{i}}$ delivered by the $i^{\text {th }}$ element of the detector when the camera was placed in front of the black body source at different temperatures $T$,

$$
s_{i}(T)=\sum_{p=0}^{P} a_{i p} T^{p} \text { for } T \in\left[T_{1}, T_{2}\right]
$$

The $a_{i p}$ coefficients are derived from a least-squares fit. The user may first define the degree $P$ of the polynomial fitting function. Generally polynomials of degree $P \geq 5$ are chosen. The user defines a number $J$ of equilibrium thermal states $T_{j}$ distributed between $T_{1}$ and $T_{2}$. 
The calibration protocol is partly managed by computer. Once the data are fitted, defective pixels are detected using the following criterion. The system will consider pixel $i$ as a bad one if the difference between the temperature $T_{i j}$ (predicted by the polynomial fitting) and the ordered temperature of the black body source $T_{\mathrm{j}}^{B B}$ is greater than a predefined threshold $\delta T$. This threshold is typically about $\delta T=40 \mathrm{mK}$ and must be greater than the black body specifications. This criterion can be written as:

$$
\sup _{j=1,2, . ., J}\left|T_{\mathrm{ij}}-T_{\mathrm{j}}^{B B}\right| \geq \delta T
$$

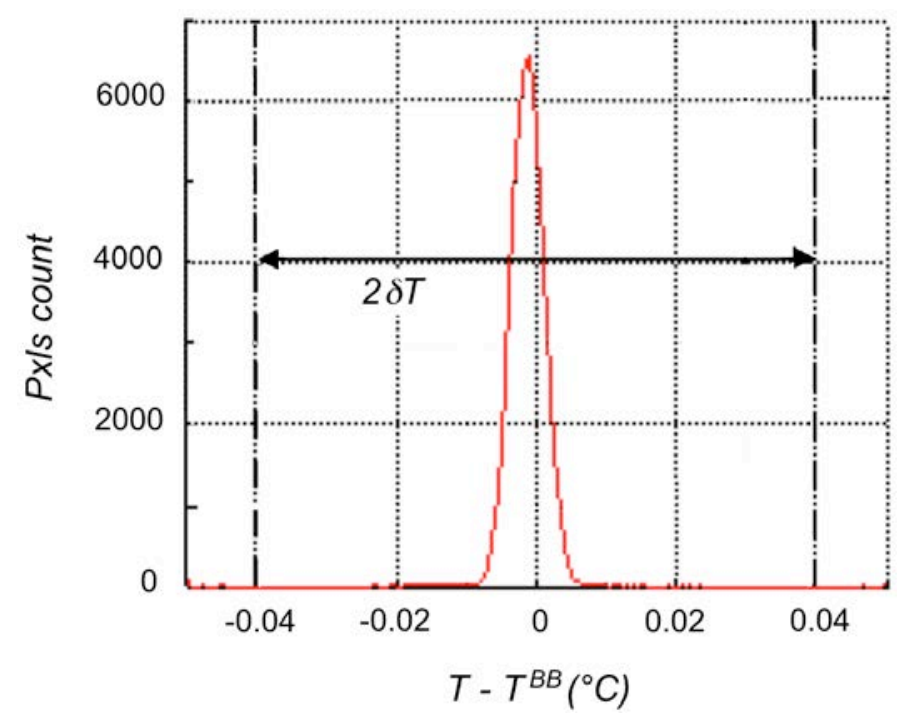

Figure 3. Histogram of pixel temperature differences at fixed $\mathrm{T}^{\mathrm{BB}}\left(17^{\circ} \mathrm{C}\right)$. Integration time IT $=2 \mathrm{~ms}$, full frame, focal length $=25 \mathrm{~mm}$, after [23].

To illustrate this criterion, Figure 3 shows a histogram characterizing the response of the pixel matrix for a given black body temperature $T^{B B}$. It indicates the number of pixels giving the same difference $\left(T_{\mathrm{ij}}-T^{B B}\right)$. This error depends on:

- the temperature range $\left[T_{1}, T_{2}\right]$ used in the fitting operation

- the degree $P$ of the polynomial fitting function 
- the camera parameter settings: integration time, frame size, focal length, etc.

The pixel response distribution looks like a quasi-centered Gaussian law. The number of pixels whose response is outside of the threshold band is low. For example, in the case of Figure 3, 0.2\% of bad pixels were counted.

\subsubsection{Heat source estimate}

The 3D heat diffusion equation makes a direct link between thermal and calorimetric aspects of the deformation mechanisms. Indeed, in the framework of quasi-static tests and under some hypotheses (e.g. constancy of thermophysical parameters), Equation (2) contains linear partial differential operators applied to temperature in the left hand side, with the different heat sources being grouped in the right hand side. From a mathematical standpoint, the determination of heat sources when the surface (i.e. boundary) temperature distribution is known is a so-called inverse problem and is very difficult, often impossible, to solve because of the smoothing effects of the heat diffusion and the lack of prior information on the heat source distribution itself.

This is why our first goal was to get a simplified heat equation, adapted to thin, flat specimens, so as to be able to compute $2 \mathrm{D}$ distributions of heat sources using surface thermal field measurements. We therefore considered an averaged diffusion equation over the sample thickness "to convert" surface temperatures into mean heat sources.

Then, by integrating the heat equation (Equation (2)) over the sample thickness [24], and defining the mean thermal disequilibrium over the thickness between the sample and its surroundings by $\bar{\theta}$, the following $2 \mathrm{D}$ diffusion equation can be obtained:

$$
\rho C \dot{\bar{\theta}}-k\left(\frac{\partial^{2} \bar{\theta}}{\partial x^{2}}+\frac{\partial^{2} \bar{\theta}}{\partial y^{2}}\right)+\rho C \frac{\bar{\theta}}{\tau_{t h}}=\bar{w}_{\mathrm{h}}^{\circ},
$$


where $x$ and $y$ are in-plane coordinates. The parameter $\tau_{\text {th }}$ represents a time constant characterizing heat losses by convection and radiation between the sample surfaces and the surroundings. In accordance with the plane stress assumption (negligible sample thickness), and the high thermal diffusivity of the tested material, the depth-wise averaged temperatures remain close to the surface temperatures. The mean temperature variation $\bar{\theta}$ is then identified with the surface temperature given by the IR camera. Moreover, $\dot{\bar{\theta}}=\partial \bar{\theta} / \partial t+v \cdot \operatorname{grad} \bar{\theta}$ is the specific time derivative of $\bar{\theta}, v$ representing the velocity vector. In the case of localized flow with strong thermal gradients, the time derivative should take the convective term $v \cdot \operatorname{grad} \bar{\theta}$ into account.

To calculate such convective terms, we showed that it is possible to combine kinematic and thermal data, used in parallel with infrared thermography (IRT), digital image correlation (DIC) techniques [20,21].

The computation of heat sources then requires the estimation of different partial derivatives of the surface temperature. Although this computation no longer corresponds to an ill-posed inverse problem, it still remains tricky from a numerical standpoint. Indeed, derivatives have to be locally estimated using discrete and noisy thermal data. In particular, correct computation of the $2^{\text {nd }}$ order space derivatives (Laplacian term) is actually difficult. To reduce the noise effect, amplified by the many derivations, filtering or fitting tools can be considered.

Because of the slowness of the first digitization systems of monodetector cameras used in the 1990s (typically two digitized images per second with the famous Intel 80286 processor), Fourier's techniques were essentially limited to spatial filtering in the image processing [25]. Convolutive filtering using the discrete Fourier transform were especially used [24]. The main drawbacks of these techniques were: 
- A previous periodic expansion of thermal images, preferable before any heat source computation. This expansion naturally requires a lot of memory space and is time consuming. It is however necessary to avoid supplementary frequencies coming from the automatic periodization induced by the discrete Fourier transform. We showed that these additional frequencies may considerably disturb the computation of heat sources using convolutive filtering.

- The impossibility of reducing all noise effects, with the thermal noise being centred white noise with a uniform power spectrum. We used a low-pass Gaussian filter, which is a smooth filter even when it is twice derived (Laplacian computation).

More recently, fitting techniques were developed with IRFPA cameras. Local least-squares approximation of temperature fields using a different set of approximation functions was used: a set of space-time polynomials for monotonous loading $[20,26]$, trigonometric in time and polynomial functions in space for monochromatic fatigue tests [27]. The optimized sizes of the cubic space-time approximation domain naturally have to be adjusted according to the heterogeneity of the sought heat source distribution and of the signal-to-noise ratio.

For the fitting methods, we recently estimated that the peak-to-peak thermal noise was about $200 \mathrm{mK}$ (i.e. before data fitting) and the range of the thermal noise dropped to $2 \mathrm{mK}$ for standard fitting parameters. Moreover, the order of magnitude of the spatial resolution (in terms of pixel size) was about $0.4 \mathrm{~mm}$ and the temporal resolution was considered to be equal to $0.04 \mathrm{~s}$.

An overall estimate of the uncertainty on heat source estimates should take into account every possible error source associated with: (i) the temperature accuracy, (ii) knowledge of the thermophysical parameters, (iii) the relevance of the thermal model (heat exchanges, source distribution) and its identification, (iv) mapping between 
kinematic and thermal data when convective terms have to be computed, and $(v)$ the image processing robustness (derivation of discrete, noisy temperature fields).

By limiting the error analysis to items $(i)$ and $(v)$, we estimated the uncertainty by computing the heat source distribution from a set of IR images of a non-loaded specimen. The parasitic sources derived from the image processing then gave an order of magnitude for the relative uncertainty on the heat source fields. We found an average parasitic heat source value of about $2 \times 10^{-5} \mathrm{~W} \cdot \mathrm{mm}^{-3}$ with a standard deviation of $3 \times 10^{-5}$ W. $\mathrm{mm}^{-3}$.

\section{Examples illustrating the application}

To illustrate, through application examples, the crucial importance of analyzing the thermal and calorimetric responses associated with material deformation processes, cyclic tensile tests are considered in the sequel. When a stabilized mechanical cycle occurs, the hysteresis area has three possible origins: energy dissipation, internal energy variations and thermomechanical coupling mechanisms [28]. Determining, through a calorimetric analysis of the thermal response, the origins of the hysteresis area is consequently of primary importance to model the material behavior. The dissipation reflects the irreversibility of the deformation mechanisms; the internal energy variations reveal the energy cost of microstructural evolutions and the coupling heat sources indicate that microstructural and mechanical states of the matter are highly temperature dependent.

\subsection{Thermo-elasticity vs. visco-elasticity}

The academic case of linearized thermoelasticity can be evoked as evidence of the third origin of hysteretic mechanical responses. For thermoelastic behavior, a stabilized mechanical cycle becomes a thermodynamic cycle and the only allowed source of 
irreversibility is due to heat diffusion. For simplicity, a "0D" heat equation can be considered. Indeed, 3D heat diffusion mechanisms can be bypassed assuming linear heat losses depending only on the temperature variation [28]. In such a simplified context, the main equations of the thermoelastic problem are then summed up by the following coupled algebraic and differential equations:

$$
\left\{\begin{array}{l}
\sigma=E\left(\varepsilon-\lambda_{\text {th }} \theta\right) \\
\dot{\theta}+\frac{\theta}{\tau_{\text {th }}}=-\frac{\lambda_{\text {th }} E\left(T_{0}+\theta\right) \dot{\varepsilon}}{\rho C}
\end{array}\right.
$$

where $\sigma$ is the tensile stress, $\varepsilon$ the longitudinal strain, $\tau_{\text {th }}$ a time constant characterizing the heat losses, $E$ the Young's modulus and $\lambda_{t h}$ the linear thermo-dilatation coefficient. During a cyclic strain-controlled test $\varepsilon=\varepsilon_{0} \sin \left(2 \pi f_{\mathrm{L}} t\right)$, where $\varepsilon_{0}$ is the stress amplitude and $f_{\mathrm{L}}$ the loading frequency, it can be experimentally noted, after some cycles, that the temperature variations progressively become periodic and symmetric with respect to the time axis. Once the periodic regime is reached, (cf. Figure 4) the mechanical cycles become thermodynamic cycles and the hysteresis area $A_{h}$ (i.e. the deformation energy $w_{d e f}$ over a mechanical cycle) reads:

$$
w_{\text {def }}=A_{h}=\int_{t}^{t+f_{\mathrm{L}}^{-1}} \sigma \dot{\varepsilon} \mathrm{d} \tau=\int_{t}^{t+f_{\mathrm{L}}^{-1}} w_{t h e}^{\circ} \mathrm{d} \tau=w_{t h e} .
$$

In non-adiabatic and non-isothermal situations, thermoelastic coupling then induces rate dependence. It would be interesting to highlight this rate dependence by writing a rheological equation. From equations (8a-b,) we get, by substituting the temperature, in case of small thermal variations $\left(\theta<<T_{0}\right)$ :

$$
\sigma+\tau_{\text {th }} \dot{\sigma} \approx E \varepsilon+E \tau_{\text {th }}\left(1+\frac{E \lambda_{\text {th }}^{2} T_{0}}{\rho C}\right) \dot{\varepsilon} .
$$




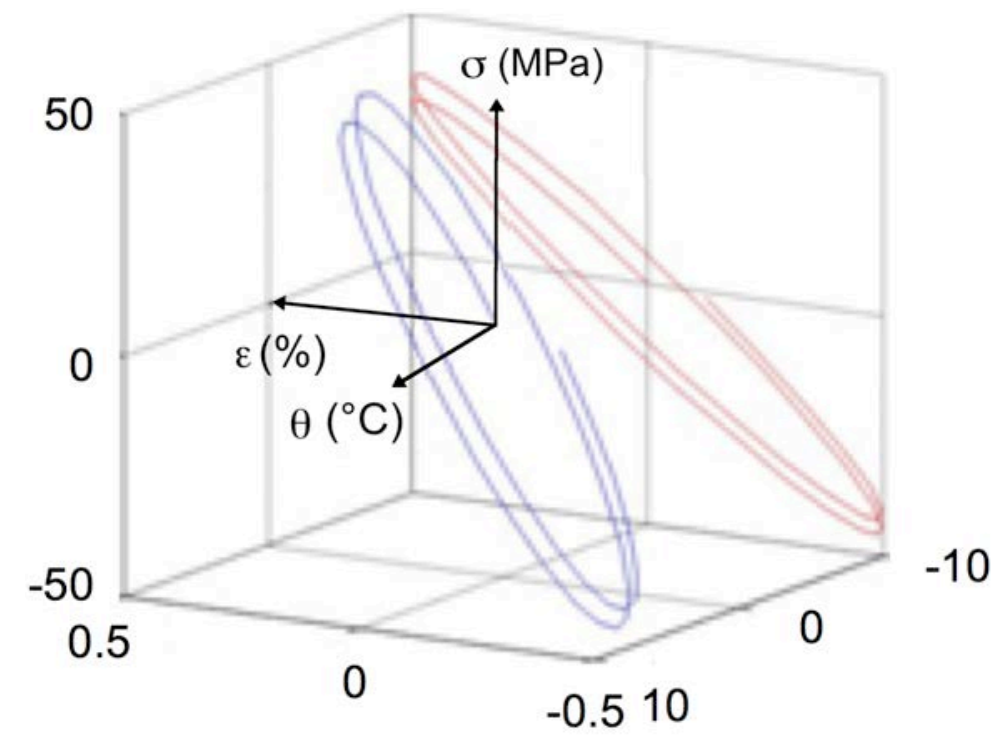

Figure 4. Thermoelastic cyclic responses; the typical values chosen for the simulation are close to polymer characteristics: $\mathrm{E}=1000 \mathrm{MPa}, \rho=1000 \mathrm{~kg} \cdot \mathrm{m}^{-3}, \mathrm{C}=1000 \mathrm{~J} \cdot \mathrm{kg}^{-1} \cdot{ }^{\circ} \mathrm{C}^{-1}, \tau_{\text {th }}=20 \mathrm{~s}$. The thermodilatation coefficient was multiplied by $100\left(\lambda_{\text {th }}=5.10^{-3}{ }^{\circ} \mathrm{C}^{-1}\right)$ to amplify the hysteresis effect induced by coupling and heat diffusion. Loading parameters were: $\mathrm{f}_{\mathrm{L}}=0.05 \mathrm{~Hz}, \varepsilon_{0}=$ 0.005 .

This rheological equation looks like those obtained in linear (isothermal!) viscoelasticity. This similarity naturally complicates the behavior analysis, particularly if the thermoelastic effects are, from a calorimetric standpoint, greater than the viscous effects. For such materials, the results, given by standard visco-analysis and leading to the determination of complex moduli, are harder to interpret because of the overlap of two similar independent time effects respectively induced by viscous dissipation and thermoelastic coupling.

For example, PMMA and PC polymers, very often used in industrial applications, have been considered by [29]. The temperature corresponding to the first sub-vitreous relaxation peak of the PMMA was slightly greater than the room temperature at which the tests were performed $\left(T_{0} \approx 20-24^{\circ} \mathrm{C}\right)$, while the relaxation peak temperature of the PC polymer was highly negative. Consequently, at around room 
temperature, the PC samples showed greater molecular mobility than the PMMA samples.

Due to this property, the PC samples showed good ductility while the PMMA samples remained particularly brittle. As the glass transition temperature of both polymers is greater than $100^{\circ} \mathrm{C}$, they naturally remained in a glassy state during the deformation tests. The main thermo-mechanical characteristics of both materials are grouped in Table 2.

Table 2 Main thermophysical properties of PMMA and PC.

\begin{tabular}{cccc}
\hline & Origin & PMMA & PC \\
\hline$\rho\left(\mathrm{Kg} \cdot \mathrm{m}^{-3)}\right.$ & Measured & 1160 & 1190 \\
$C\left(\mathrm{~J} . \mathrm{Kg}^{-1} \cdot{ }^{\circ} \mathrm{C}^{-1}\right)$ & Atofina & 1450 & 1200 \\
$k\left(\mathrm{~W} \cdot \mathrm{m}^{-1} \cdot \mathrm{K}^{-1}\right)$ & Atofina & 0.17 & 0.2 \\
$\lambda_{\text {th }} 10^{6}\left({ }^{\circ} \mathrm{C}^{-1}\right)$ & Measured & 7 & 7 \\
$E(\mathrm{MPa})$ & Measured & 3650 & 2650 \\
\hline
\end{tabular}

Standard dog bone shaped test specimens were used with the following gauge part sizes: length $(60 \mathrm{~mm})$, width $(10 \mathrm{~mm})$, and thickness $(4 \mathrm{~mm})$.

Figures 5a and 6a show the stabilized stress-strain hysteresis loop for both materials during tests at a constant absolute strain rate (about $10^{-4} \mathrm{~s}^{-1}$ ), with a constant stress range. The primacy of the thermoelastic effects over the dissipation can then easily be suspected due to the temperature variation. We observed a distinct cooling of the specimen during the loading stages and heating during the unloading stages, with the mean temperature over each load-unload cycle being approximately zero. 


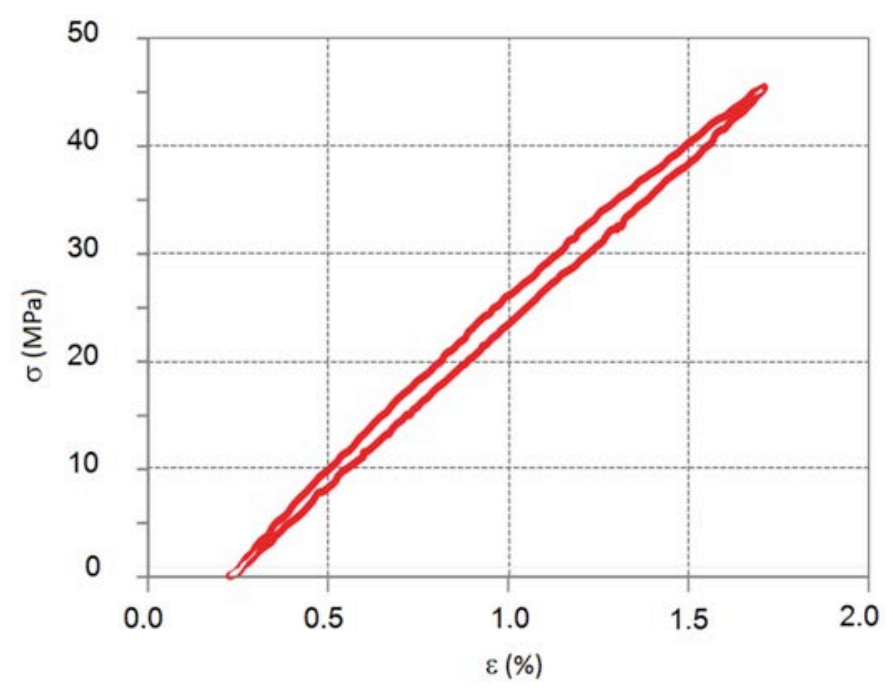

a

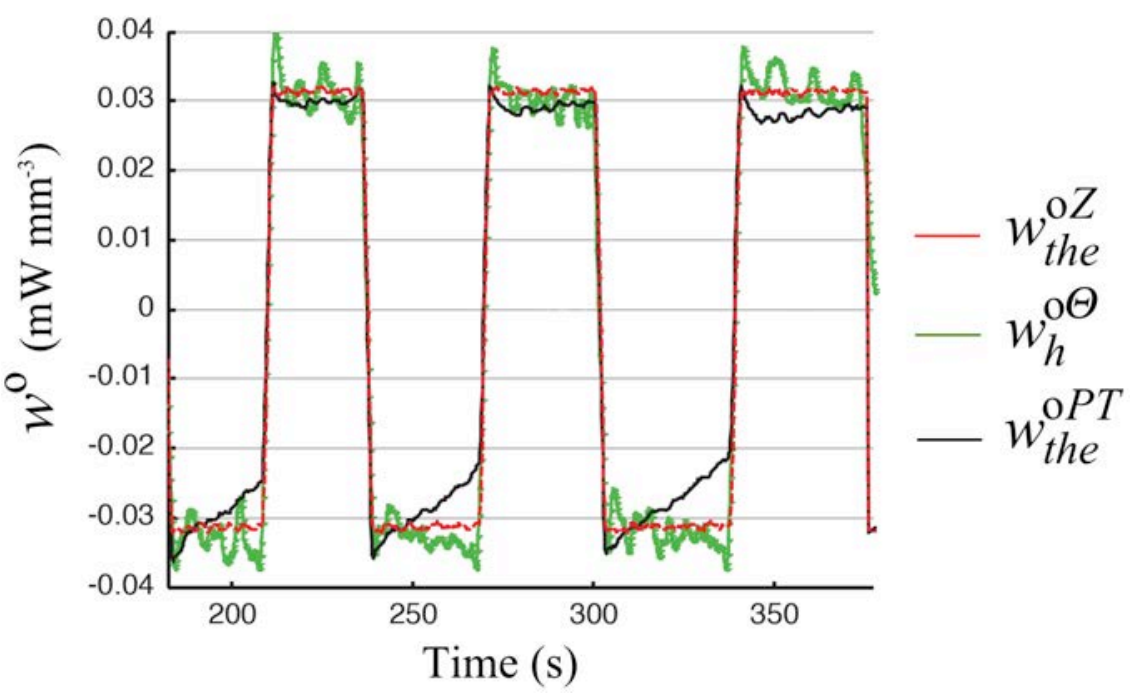

Figure 5. (a) example of a stabilized stress-strain response (PMMA); (b) primacy of thermoelastic effects $w_{h}^{\mathrm{O} \Theta}, w_{\text {the }}^{\mathrm{o} Z}, w_{\text {the }}^{\mathrm{O} P T}$ patterns associated with load-unload cycles with increasing stress ranges and a constant absolute strain rate value $\left(|\mathrm{d} \varepsilon / \mathrm{dt}|=4.3510^{-4} \mathrm{~s}^{-1}\right)$.

Figures $5 \mathrm{~b}$ and $6 \mathrm{~b}$ show the time variation of the overall heat sources $w_{h}^{\mathrm{o}}$ derived from the IR thermal data during cycles performed at a constant (absolute) strain rate with an increasing stress range. They confirmed the negligible character of the dissipation. We also plotted the numerical predictions of thermelastic sources given by Zener and Poynting-Thomson type models, where the elastic branches were replaced by thermoelastic ones [29]. We then noticed that the series and parallel viscoelastic models were no longer equivalent when thermoelastic effects were taken into account. 


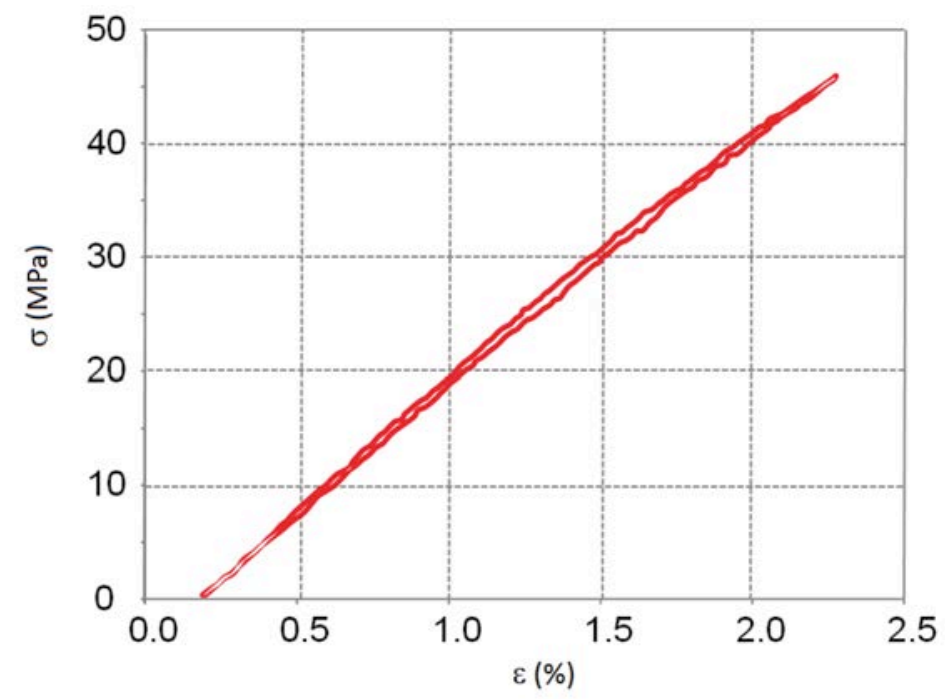

a

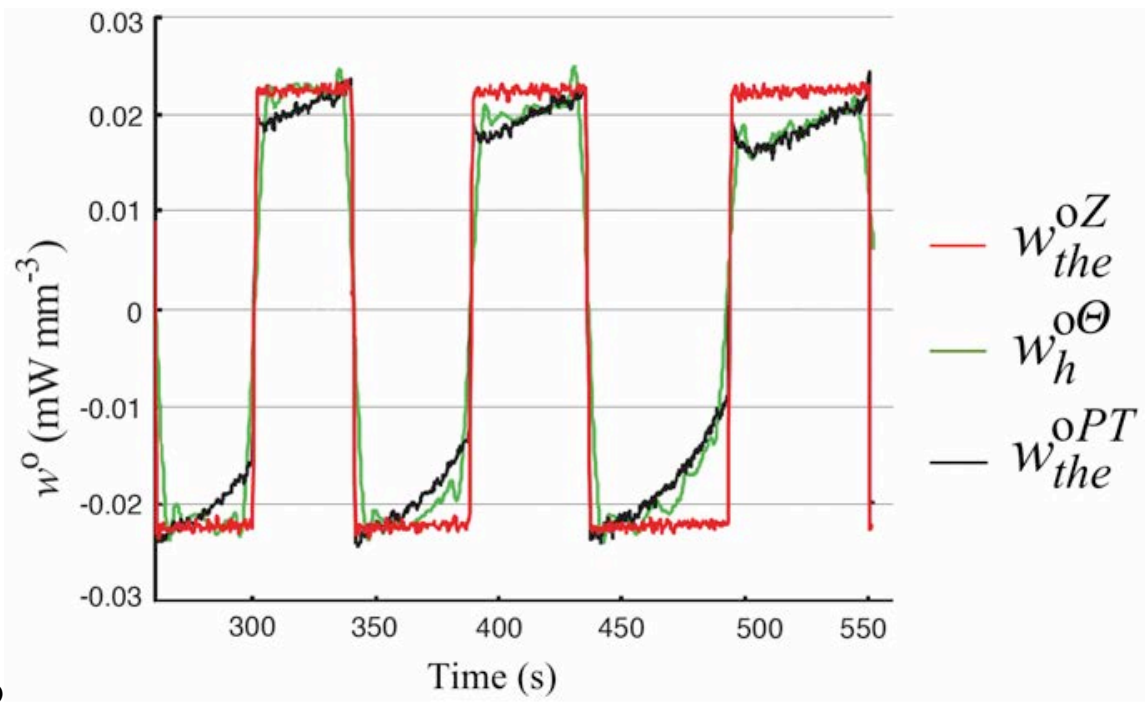

Figure 6. (a) Example of a stabilized stress-strain response (PC); (b) primacy of thermoelastic effects $w_{h}^{\mathrm{o} \Theta}, w_{\text {the }}^{\mathrm{o} Z}, w_{\text {the }}^{\mathrm{o} P T}$ patterns associated with load-unload cycles with increasing stress ranges and a constant absolute strain rate $\left(|\mathrm{d} \varepsilon / \mathrm{dt}|=4.3510^{-4} \mathrm{~s}^{-1}\right)$.

Regarding the PMMA behavior, Figure 5b shows that variations in $w_{h}^{\mathrm{o} \Theta}$ and $w_{\text {the }}^{\mathrm{oZ}}$ remained close at all stress levels. Conversely, the source $w_{h}^{\mathrm{o} \Theta}$ was quasi linear throughout the loading, while $w_{h}^{\mathrm{\Theta}}$ remained almost constant. Regarding the PC behavior, estimates of the same sources were plotted in Figure 6b. A better prediction was obtained with the PT type model. 
For these two studied materials, it should be noted that the order of magnitude of the thermoelastic source amplitude was about $60 \mu \mathrm{W} \cdot \mathrm{mm}^{-3}$ for a dissipation intensity of less than $2 \mu \mathrm{W} \cdot \mathrm{mm}^{-3}$, which is the order of magnitude of heat sources corresponding to the thermal noise.

\subsection{Fatigue of steel}

The approximation underlying the existence of an elastic domain becomes unsound when a large number of "elastic" cycles are performed. Indeed, for crystalline materials, dislocations slightly oscillate even under low cyclic stress amplitudes. Cycle by cycle, dislocations gradually gather to build up persistent slip bands (PSB), which are microscopic zones where the plasticity preferentially develops inside some "well" oriented grains [30]. This microplasticity development finally leads to microcracks followed by a sudden rupture. This is the so-called high cycle fatigue (HCF) of materials and it has become essential to master the kinetics of this phenomenon in industrial applications for obvious economical and safety reasons. The HCF analysis of materials and structures currently involves a large number of research centers throughout the world. We recently showed that the thermomechanical approach could be beneficially applied to gain further insight into the fatigue kinetics. From an image processing standpoint, the main difficulty was to extract low dissipation intensities from large thermoelastic source amplitudes.

Readers interested in the use of quantitative infrared imaging techniques applied to the analysis of fatigue mechanisms can refer to [31,32] where the IR image processing developed to process fatigue tests is detailed. Results of applications to steel and aluminum can be found in [33, 34] and more recently in [32] where energy balance distributions were constructed using DIC and IRT techniques. To briefly illustrate the 
potential of IR image analysis, Figure 7 shows the mean dissipation pattern per cycle $\tilde{d}_{1}$ calculated at different stress ranges $\Delta \sigma$. During a block of 20000 cycles, this heterogeneous pattern remains relatively constant as long as a fatigue crack does not initiate.
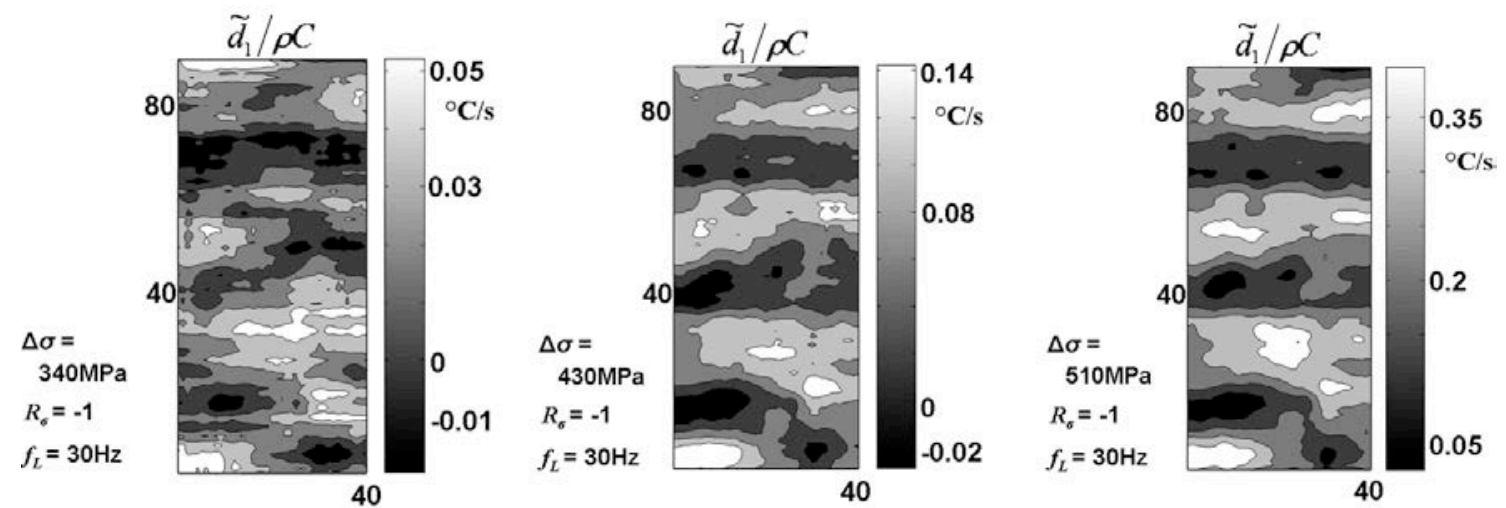

Figure 7. Dissipation intensity versus stress range; from [34]; (volume heat source divided by $\rho \mathrm{C}$ are expressed in $\left.{ }^{\circ} \mathrm{C} \cdot \mathrm{s}^{-1}\right)$

The contour plots in Figure 7 also show that areas where the dissipation is high (respectively low) remain approximately the same from one block to another, even though the dissipation naturally increases with the stress range. During a (last) loading block, we observed a localized zone of high dissipation several hundreds of cycles before crack onset. Moreover, we also noticed that this zone always corresponded to a candidate zone where the dissipation level was high from the beginning of the fatigue test (i.e. for low stress ranges). 


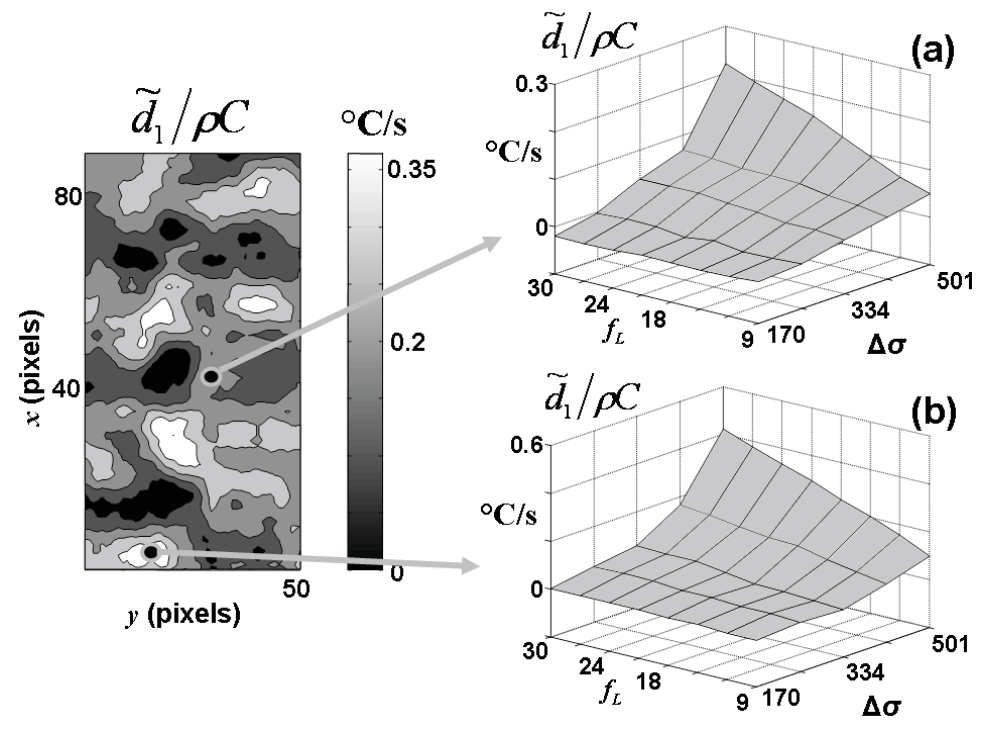

Figure 8. Dissipation patterns within two different material zones; from [34].

Besides, Figure 8 shows examples of dissipation variations associated with zones of the same specimen as a function of $\Delta \sigma$ and $f_{L}$. During this test performed at $R_{\sigma}=-1, \Delta \sigma$ increased from 170 to $500 \mathrm{MPa}$ and $f_{L}$ increased from 9 up to $30 \mathrm{~Hz}$ for each load level. Dissipation values in the first location (Figure 8a) are twofold lower than that measured in the second area (Figure $8 \mathrm{~b})$. First, the shape of both surfaces $\tilde{d}_{1}\left(\Delta \sigma, f_{\mathrm{L}}\right)$ is clearly very similar. At constant stress, we also underline that $\tilde{d}_{1}$ increases in a quasilinear way with the loading frequency $f_{L}$ for both zones and for all stress ranges. This linearity of $\tilde{d}_{1}$ with $f_{L}$ is consistent with an interpretation of the fatigue mechanisms in terms of time-independent microplasticity.

\section{Concluding comments}

The potential applications of infrared thermography are likely to have a much broader relevance to the mechanics of materials than what we illustrate here. The thermomechanical behavior of material under mechanical and thermal loading is too 
often assessed by identifying the material temperature and comparing it to that of the ambient environment (e.g. room temperature). When its role is not reduced to that of a parameter, the temperature is often referred to as a control variable. These approximations surely mask important phenomena that cannot be identified without adopting a true thermomechanical approach to the material behavior. Advances in thermographic imaging now enable access to quantitatively reliable temperature measurement fields. These fields can then be used to assess certain physical coefficients such as local diffusivity or conduction of the studied material. In the near future, these distributions of thermophysical properties could be used to detect material irregularities that are natural or induced by mechanical and thermal loadings. Once these thermophysical characteristics are known, temperature fields can then be used to estimate heat sources accompanying the deformation, while reflecting the irreversibility of the deformation mechanisms and/or thermomechanical coupling effects.

The example mentioned here was limited to quasi-static transformations at constant temperature. Although much remains to be done, it should not be considered that this is the only application area of infrared techniques. Increasingly, thermographic analysis can address the problem of modeling the behavior of materials at high temperatures and dynamics. Indeed, during physical treatment (hardening, annealing, etc.), or welding processes (melting, solidification), thermomechanical coupling phenomena (phase transitions, precipitation kinetics) are of critical importance. The constant development of faster IR cameras already allows mechanical engineers to address the dynamic behavior of materials in which, again, couplings play an important role in the instability propagation mode (i.e. adiabatic shear bands, auto-catalytic cycles). 


\section{References}

[1] Thomson W (Lord Kelvin). On the thermoelastic and thermomagnetic properties of matter. Trans. Roy. Soc. Edinb. 1853; 20 (161): 57-77.

[2] Thomson W (Lord Kelvin). On the dynamical theory of heat, with numerical results deduced from Mr Joule's equivalent thermal unit. Quart. J. of Pure and Appl. Math. 1857; 1 (57): 261-288.

[3] Dulieu-Barton JM, Eaton-Evans J, Little EG, Brown IA. Thermoelastic analysis of high pressure angioplasty balloons. Strain. 2008; 44 (1): 102-118.

[4] Sathon N, Dulieu-Barton JM. Evaluation of sub-surface stresses using thermoelastic stress analysis. Applied Mechanics and Materials. 2007; 7-8: 153158.

[5] Luong MP. Fatigue limit evaluation of metals using an infrared thermographic technique. Mechanics of Materials. 1998; 28:155-163.

[6] La Rosa G, Risitano A. Thermographic methodology for rapid determination of the fatigue limit of materials and mechanical components. International Journal of Fatigue. 2002; 22 (1): 65-73.

[7] Krapez J-C, Pacou D, Gardette G. Lock-in thermography and fatigue limit of metals. Proceedings of QIRT'2000 Conf., Reims, France, July 18-21, 2000: 277282. QIRT Open Archives: http://qirt.gel.ulaval.ca/dynamique/index.php?idD=45, Paper QIRT 2000-051.

[8] Doudard C, Calloch A, Hild F, Cugy P, Galtier A. Identification of the scatter in high cycle fatigue from temperature. C.R. Acad. Sci. 2004; 232 (10): 785-801.

[9] Wagner D, Ranc N, Bathias C. Paris PC. Fatigue crack initiation detection by an IR thermography method. Fatigue \& Fracture of Engineering Materials \& Structures. 2010; 33 (1): 12-21.

[10] Fargione G, Geraci A, La Rosa G, Risitano A. Rapid determination of the fatigue curve by the thermographic method, International Journal of Fatigue. 2002; 24 (1): 11-19.

[11] Cugy P, Galtier A. Microplasticity and temperature increase in low carbon steels. Proceedings $8^{\text {th }}$ Int. Fatigue Conference, Stockholm. 2002: 549-556.

[12] Nayroles B, Bouc R, Caumon H, Chezeaux JC, Giacometti E. Téléthermographie infrarouge et mécanique des structures" (Infrared telethermography and structures mechanics). Int. J. Engng. Sci. 1981; 19 (7): 929-947. 
[13] Brémond P. Développement d'une instrumentation infrarouge pour l'étude des structures mécaniques. PhD thesis, Marseille University, France. 1982.

[14] Ranc N, Taravella L, Pina V, Hervé P. Temperature field measurement in titanium alloy during high strain rate loading - adiabatic shear bands phenomenon. Mechanics of Materials. 2008; 40 (4-5): 255-270.

[15] Sutter G, Ranc N.Temperature fields in a chip during high-speed orthogonal cutting - An experimental investigation. Int. J. of Machine Tools \& Manufacture. 2007; 47: 1507-1517.

[16] Mandel J. Energie élastique et travail dissipé dans les modèles (Elastic energy and dissipated work in models). Cahiers du Groupe Français de Rhéologie. 1965; I (1): 9-14.

[17] Bui HD. Dissipation d'énergie dans une déformation plastique (Energy dissipation in a plastic deformation). Cahiers du Groupe Français de Rhéologie. 1965; I (1): 15-19.

[18] Pron H, Bissieux C. Focal plane array camera as research tools. Quantitative Infrared Thermography Journal. 2004; 1 (2): 229-240.

[19] Batsale J-C, Le Niliot C. Estimation de paramètres par thermographie IR (Parameter estimate by IR thermography). In: Métrologie Thermique et Techniques inverses (Thermal metrology and inverse techniques). Ed. Presses Universitaires de Perpignan. 2001; 1, chap.4: 179-236.

[20] Chrysochoos A, Wattrisse B, Muracciole J-M, El Kaïm Y. Fields of stored energy associated with localized necking of steel. J. of the Mechanics of Materials and Structures. 2009; 4 (2): 245-262.

[21] Wattrisse B, Muracciole J-M, Chrysochoos A. Thermomechanical effects accompanying the localized necking of semi-crystalline polymers. Int. J. of. Therm. Sci. 2001; 41: 422-427.

[22] Pappini F, Gallet P. Thermographie infrarouge - Image et mesure (Infrared thermography - Image and measurement). Ed. Masson. 1997.

[23] Honorat V, Moreau S, Muracciole JM, Wattrisse B, Chrysochoos A. Calorimetric analysis of polymer behaviour using a pixel calibration of an IRFPA camera. Quantitative Infrared Thermography journal. 2005; 2 (2): 153-172.

[24] Chrysochoos A, Louche H. An infrared image processing to analyze the calorific effects accompanying strain localization. Int. J. Eng. Sci. 2000; 38: 1759-1788. 
[25] Chrysochoos A. Energy-balance for elastic-plastic deformation at finite strain. J. Mec. Théo et Appl. 1985; 4 (5): 589-614.

[26] Wattrisse B, Chrysochoos A, Muracciole J-M, Nemoz-Gaillard M. Analysis of strain localization during tensile test by digital image correlation. J. Exp. Mech., 2000; 41 (1): 29-38.

[27] Berthel B, Chrysochoos A, Wattrisse B, Galtier A. Infrared image processing for the calorimetric analysis of fatigue phenomena. Experimental Mechanics. 2008; 48 (1): 79-90.

[28] Chrysochoos A, Huon V, Jourdan F, Muracciole J-M, Peyroux R, Wattrisse B. Use of Full-Field DIC \& IRT Measurements for the Thermomechanical Analysis of Material Behaviour. Strain. 2010; 46:117-130.

[29] Moreau S, Chrysochoos A, Muracciole J-M, Wattrisse B. Analysis of thermoelastic effects accompanying the deformation of PMMA and PC polymers. C. R. Acad. Sci. 2005; 333 (8): 648-653.

[30] Mughrabi H. Dislocation wall and cell structures and long range internal stresses in deformed metal crystals. Acta Metallurgica. 1983; 31 (9): 1367-1379.

[31] Boulanger T, Chrysochoos A, Mabru C, Galtier A. Calorimetric and thermoelastic effects associated with the fatigue behavior of steels. Int. J. of Fatigue. 2004; 26: 221-229.

[32] Chrysochoos A, Berthel B, Latourte F, Pagano S, Wattrisse B, Weber B. Local energy approach to fatigue of steel. Strain: Int. J Exp. Mech. 2008; 44: 327-334.

[33] Morabito AE, Chrysochoos A, Dattoma V, Galietti U. Analysis of heat sources accompanying the fatigue of 2024 T3 aluminium alloys. Int. J. of Fatigue. 2007; 29 (5): 977-984.

[34] Berthel B, Galtier A, Wattrisse B, Chrysochoos A. Thermographic analysis of fatigue dissipation properties of DP60 steel. Strain: Int. J Exp. Mech. 2007; 43: 273-279. 